\title{
Altered expression of parvalbumin immunoreactivity in rat main olfactory bulb following pilocarpine-induced status epilepticus
}

\author{
Yeon Hee Yu, Dae-Kyoon Park, Dae Young Yoo ${ }^{*} \mathcal{E}$ Duk-Soo Kim ${ }^{*}$ \\ Department of Anatomy, College of Medicine, Soonchunhyang University, Cheonan 31151, Korea
}

Epilepsy is a chronic neurological disease characterized by spontaneous recurrent seizures and caused by various factors and mechanisms. Malfunction of the olfactory bulb is frequently observed in patients with epilepsy. However, the morphological changes in the olfactory bulb during epilepsy-induced neuropathology have not been elucidated. Therefore, in the present study, we investigated the expression of parvalbumin (PV), one of the calcium-binding proteins, and morphological changes in the rat main olfactory bulb (MOB) following pilocarpine-induced status epilepticus (SE). Pilocarpine-induced SE resulted in neuronal degeneration in the external plexiform layer (EPL) and glomerular layer (GL) of the MOB. PV immunoreactivity was observed in the neuronal somas and processes in the EPL and GL of the control group. However, six hours after pilocarpine administration, PV expression was remarkably decreased in the neuronal processes compared to the somas and the average number of PV-positive interneurons was significantly decreased. Three months after pilocarpine treatment, the number of PV-positive interneurons was also significantly decreased compared to the 6 hour group in both layers. In addition, the number of NeuN-positive neurons was also significantly decreased in the EPL and GL following pilocarpine treatment. In double immunofluorescence staining for PV and MAP2, the immunoreactivity for MAP2 around the PV-positive neurons was significantly decreased three months after pilocarpine treatment. Therefore, the present findings suggest that decreases in PV-positive GABAergic interneurons and dendritic density in the MOB induced impaired calcium buffering and reciprocal synaptic transmission. Thus, these alterations may be considered key factors aggravating olfactory function in patients

*Corresponding authors. Duk-Soo Kim, Tel: +82-41-570-2471; Fax: +82-41-574-1770; E-mail: dskim@sch.ac.kr; Dae Young Yoo, Tel: +82-41-570-2472; Fax: +82-41-574-1770; E-mail: dyyoo@sch.ac.kr

https://doi.org/10.5483/BMBRep.2020.53.4.002

Received 3 January 2020, Revised 24 January 2020, Accepted 12 March 2020

Keywords: Epilepsy, Neurodegeneration, Olfaction, Parvalbumin, Rat main olfactory bulb with epilepsy. [BMB Reports 2020; 53(4): 234-239]

\section{INTRODUCTION}

Epilepsy is a chronic neurological disorder characterized by spontaneous recurrent seizures. Status epilepticus (SE) is a seizure that persists for 20 to 30 minutes or repeats without recovery (1). About $1-2 \%$ of people suffer from various epileptic diseases and treatment is not effective for $20-40 \%$ of the patients with epilepsy (2). In addition, previous investigators have reported excitotoxic injury, necrosis, and programmed apoptotic cell death in the brain induced by epilepsy (3). In the mouse pilocarpine-induced SE model massive neurodegenerative lesions were detected in the brain subregions including the hippocampus and cerebral cortex (4). Behavioral and cognitive disorders have been observed in epileptic animal models, as well as in patients, as a result of seizure-induced neuronal damage $(5,6)$. Moreover, cognitive impairment by significant olfactory dysfunction was frequently detected in patients with temporal lobe epilepsy (7).

The olfactory circuitry has been studied for over a hundred years and its well-defined structure and connections have been recently clarified (8). The olfactory bulb has a highly layered structure containing dendrodendritic synapses of various neurons and is regarded as a favorable region for morphological, electrophysiological and biochemical analyses (9). In a previous study, parvalbumin (PV) immunoreactivity was detected in the $\gamma$-aminobutyric acid (GABA)ergic interneurons in the external plexiform layer (EPL) and glomerular layer $(\mathrm{GL})$ of the main olfactory bulb (MOB) and PV expression was decreased with aging (9). Calcium-binding proteins, including PV, modulate $\mathrm{Ca}^{2+}$ concentrations that are crucial for diverse cellular events, such as synaptic transmission, cell division, neurite outgrowth and neurodegeneration (10-12). In the nervous system, PV is known to be expressed on fast-spiking GABAergic interneurons (13) and PV-positive interneurons are vital for normal olfactory function by regulating principal cells in the MOB (14). However, although many studies have focused on the connections of the olfactory circuitry and its functional odor tuning, little is known about the expression of calcium-binding proteins and morpho- 
logical changes in PV-positive interneurons in pathological states such as SE. Therefore, in the present study, we investigated the expression of PV in the subregions of the main olfactory bulb to identify a correlation between morphological changes in PV-positive interneurons and the functional loss of olfaction following SE.

\section{RESULTS}

\section{Neuronal degeneration in the MOB after pilocarpine administration}

To evaluate the neuronal degeneration in the pilocarpine-induced SE model, we performed Fluoro-jade b (FJB) staining in the control and pilocarpine-induced SE rat groups. The structure of the main olfactory bulb is described in Fig. 1A (George Paxinos and Charles Watson) and we focused on changes in FJB staining in the EPL and GL. In the control group, a few FJB-positive cells were detected in the EPL and GL of the MOB (Fig. 1B1 and C1). However, six hours after pilocarpine treatment, the number of FJB-positive cells was significantly increased in both the EPL and GL zones of the MOB compared to the control group (Fig. 1B2, C2, D and E). In addition, the elevated numbers of FJB-positive cells were still present at three months, as the period of recurrent seizures, after the induction of SE by pilocarpine treatment (data not shown).

\section{Altered PV immunoreactivity in the MOB following SE}

Immunohistochemistry for PV was performed to identify the expression and morphological changes in PV-positive interneurons in the EPL and GL of the MOB. In the control group, PV immunoreactivity was observed in neuronal somas and processes

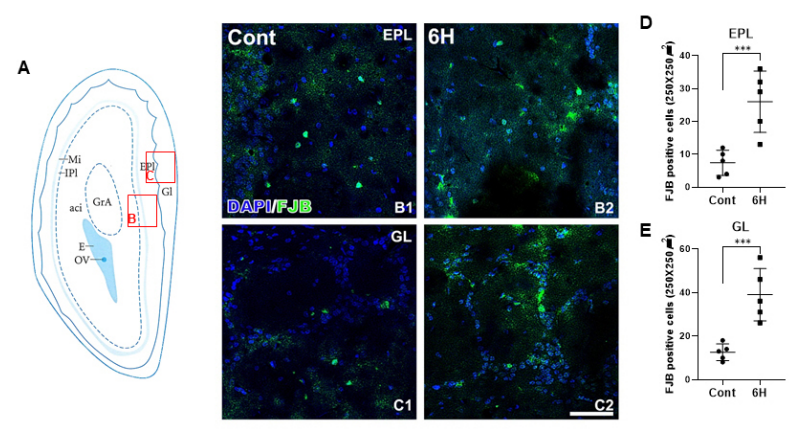

Fig. 1. Structure of the rat main olfactory bulb (MOB) adapted from the Rat Brain in Stereotaxic Coordinates (George Paxinos and Charles Watson) (A). Neuronal degeneration in the EPL (B) and GL (C) of the MOB in the control and $6 \mathrm{~h}$ after SE. The FJB positive cells expression is significantly increased in the $E P L$ and $G L$ regions compared to the control ( $\mathrm{D}$ and $\mathrm{E}$ ). All data are presented as mean \pm SEM. ${ }^{* * * P}<0.005$ vs. control. glome-rular layer; GL, external plexiform layer; $\mathrm{EPL}$, mitral cell layer; $\mathrm{Mi}$, internal plexiform layer; IPI, granule cell layer of accessory lofactory bulb; GrA, H; hours, $M$; months. Scale bar $=17 \mu \mathrm{m}$. and the average number of PV-positive interneurons was 16.75 per $250 \times 250 \mu \mathrm{m}^{2}$ in the EPL (Fig. 2A1 and 2D1). However, PV immunoreactivity in the 6 hour group after pilocarpine was remarkably decreased in the neuronal processes rather than in the somas, and the average number of PV-positive interneurons was reduced in the EPL compared to the control group (Fig. 2B1, D1, and E1). In addition, three months after pilocarpine treatment, the number of PV-positive interneurons was severely decreased in the EPL compared to six hours after pilocarpine treatment (Fig. 2C1, D1, and E1). Similar to the results observed in the EPL area, the expression of PV-positive interneurons in the GL gradually declined with time following the induction of SE. The distribution of PV-immunoreactive interneurons six hours following SE was remarkably decreased in the GL region of the MOB compared to the control and decreased expression was also observed in the dendritic processes (Fig. 2A2, B2, D2, and E2). Three months after pilocarpine treatment, PV immunoreactivity was barely detectable at the recurrent seizure time period following SE (Fig. 2C2, D2, and E2). Moreover, immunoblot analysis of PV expression showed results similar to the immunohistochemical data (Fig. 2F1 and F2).

\section{Effects of long-term epilepsy on NeuN and MAP2 immunoreactivity}

To identify the correlation between the neuronal loss in PV-positive and NeuN-positive cells, double immunofluorescence staining for these fast-spiking GABAergic interneurons and neuronal markers, respectively, was performed. In the control group, the PV immunoreactivity in the $\mathrm{NeuN}$-positive neurons was widespread and distinctly detected in the EPL of the MOB (Fig. 3A1 - D1). However, six hours after SE induction by pilocarpine treatment, the double-labeled expression of PV and NeuN was reduced in the EPL compared to the control levels (Fig. 3A2 - D2). The quantitative analyses of the immunofluorescence staining results for these two markers were similar (Fig. 3E1). The expression of PV and NeuN was lower after three months of recurrent seizures than six hours after pilocarpine treatment (Fig. 3A3 - D3), which was supported by the quantitative analysis of the immunofluorescence staining (Fig. 3E1). Similar to the results in the EPL following SE, in the GL, double-labeled PV- and NeuN- positive interneurons were seen in the same patterns within the EPL six hours after SE, but not in the control groups (Fig. 3A4 - D4 and A5 - D5). The quantitative analysis was consistent with the immunofluorescence data (Fig. 3E2). The PV and NeuN immunoreactive interneurons had disappeared three months following pilocarpine (Fig. 3A6 - D6) and the percentage of PV- and NeuN- positive interneurons was also downregulated (Fig. 3E2).

To identify the structural changes in the dendritic processes of the PV-positive interneurons, we performed double immunofluorescence staining with MAP2. In the control group, MAP2 was densely expressed in the dendrites of the PV-positive interneuronal population in the entire EPL and GL regions of 


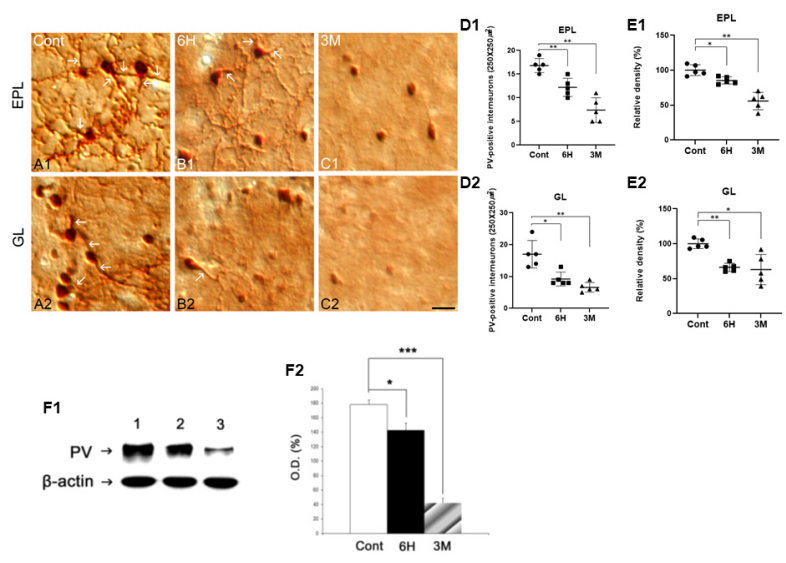

Fig. 2. Immunohistochemistry for $P V$ in the $E P L$ and $G L$ of the $\mathrm{MOB}$ in the control (A), $6 \mathrm{~h}(\mathrm{~B})$, and $3 \mathrm{M}(\mathrm{C})$ groups following SE. In the control group, PV immunoreactivity is detected in the somas and processes (A1-A2). After SE, PV immunoreactivity is markedly decreased both in the EPL and GL (B1-B2, C1-C2, D, and $\mathrm{E})$. All data are presented as mean + SEM. ${ }^{*} \mathrm{P}<0.05, * * \mathrm{P}<$ 0.01 vs. control. Glomerular layer; GL, external plexiform layer; EPL, $\mathrm{H}$; hours, $\mathrm{M}$; months. Scale bar $=17 \mu \mathrm{m}$. Western blot analysis of PV antibody in MOB following SE (F1). Lane 1, control; Lane 2, 6 hr after SE; Lane 3, 3 month after SE. Optical density (O.D.) analyses of PV protein levels in the MOB (F2). All data are presented as mean \pm SEM. ${ }^{*} \mathrm{P}<0.05,{ }^{* * *} \mathrm{P}<0.005$, vs. control. $\mathrm{H}$; hours, M; months.

the MOB (Fig. 4A1 - D1 and A4 - D4). Six hours after pilocarpine treatment, MAP2 immunoreactivity in both the EPL and GL was not changed in the PV-positive interneurons compared to the control group (Fig. 4A2 - D2 and A5 - D5). Quantitative analysis of the double immunofluorescence staining data also revealed similar results at the same time periods (Fig. $4 \mathrm{E} 1$ and $4 \mathrm{E} 2$ ). Three months after pilocarpine administration, at the recurrent seizure time period, however, the doublelabeled expression of PV and MAP2 immunoreactivity was markedly decreased in the EPL and GL (Fig. 4A3 - D3 and A6 D6). The quantitative analysis of the immunoreactivity of these two makers showed the same results as the immunostaining data at this time point (Fig. 4E1 and 4E2).

\section{DISCUSSION}

In the present study, we observed the neuronal degeneration and morphological changes following pilocarpine-induced SE in the rat MOB. Not only PV-positive interneurons but also NeuN-positive neurons were decreased time-dependently after pilocarpine treatment. Several previous studies have reported that SE resulted in oxidative stress and neuroinflammation in the brain subregions, including the hippocampus and olfactory bulb, causing seizure-induced neuronal damage $(15,16)$. In addition, functional and morphological abnormalities of the olfactory bulb were observed in the temporal lobe of epilepsy patients

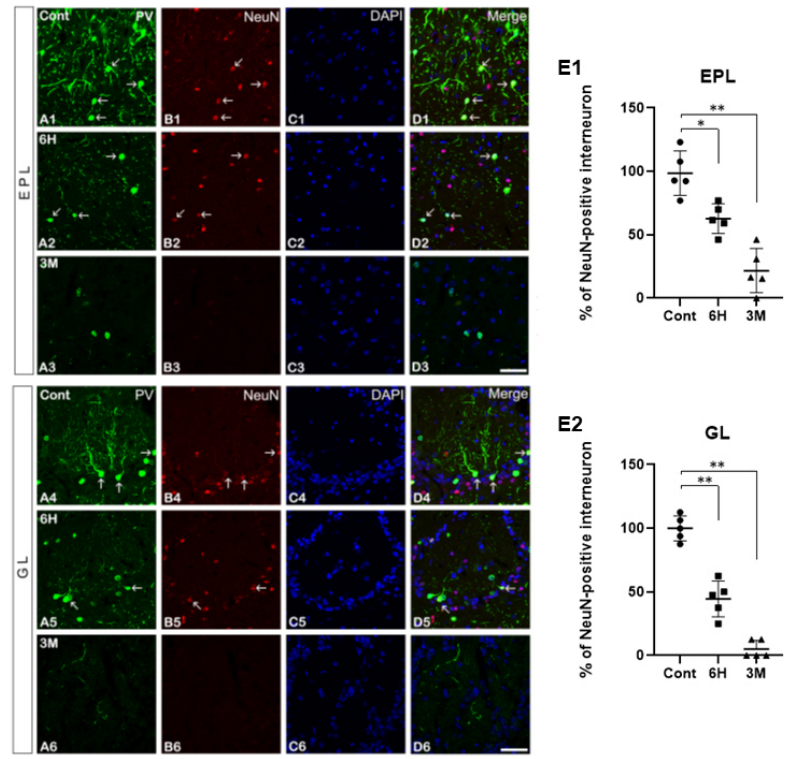

Fig. 3. Double immunofluorescence staining for $P V(A$, green), NeuN $(B$, red), with DAPI $(C)$, and merged images (D) in the EPL and GL after SE. Some of the PV-positive interneurons are co-localized with NeuN (arrows). Similar to the PV immunoreactivity pattern, the NeuN expression in the EPL and GL is significantly decreased following SE. All data are presented as mean \pm SEM. ${ }^{* P}<0.05$, $* * \mathrm{P}<0.01$ vs. control. Glomerular layer; $\mathrm{GL}$, external plexiform layer; $\mathrm{EPL}, \mathrm{H}$; hours, $\mathrm{M}$; months. Scale bar $=17 \mu \mathrm{m}$.

(7) and the olfactory bulb volumes were decreased in the temporal lobe of epilepsy patients (17). Therefore, because stimulations of the olfactory bulb can also induce seizures in a certain mouse model (18), these phenomena may support a close correlation between olfactory bulb function and epileptogenesis.

The MOB is generally known as a useful region for investigating neuronal interactions due to its distinct structures and diversity of neuroactive substances (19). Excitatory mitral and tufted cells have reciprocal dendrodendritic connections with inhibitory granule cells (20). Mitral cells receive odor input from olfactory sensory neurons and glutamate released from the excited mitral cells induces the GABAergic dendrodendritic reversion of granule cells back into mitral cells (21). In the EPL of the rat $\mathrm{MOB}, \mathrm{PV}$ is expressed in fast-spiking GABAergic interneurons $(9,13)$ and mitral cells form a reciprocal connection with these PV-positive cells. Feedback inhibition modulates the output of the mitral cells (14). These structural and functional features enable the fine-tuning of odor processing. In addition, it has been reported that PV has a crucial role in modulating intracellular calcium levels that are important for synaptic plasticity (22). In olfactory reciprocal synapses, the release of fast neurotransmitters is mediated by calcium influx through the $\mathrm{N}$ - and P/Q-type calcium channels and the magnitude of the dendrodendritic transmission depends on the level 




Fig. 4. Double immunofluorescence staining for PV (A, green), MAP2 $(B$, red), with DAPI $(C)$, and merged images $(D)$ in the EPL and GL after SE. In the control group, MAP2 expression shows dense dendritic trees (B1 and B4). Three months following SE, however, MAP2 expression is remarkably decreased compared to the early time period (B3 and B6). Relative density for MAP2 in the EPL and GL $(\mathrm{E})$. All data are presented as mean \pm SEM. ${ }^{* * P}<0.01$ vs. control. Glomerular layer; GL, external plexiform layer; EPL, $H$; hours, M; months. Scale bar $=17 \mu \mathrm{m}$.

of intracellular calcium influx (21). In the SE model of this study, as the level of PV expression decreased, we predicted that decreased calcium buffering capability and impaired inhibitory postsynaptic potential occurred, which consequently resulted in olfaction dysfunction. PV is also involved in the phosphorylation of protein kinase which has a crucial role in cell signal transduction via modulating $\mathrm{Ca}^{2+}$ and $\mathrm{Mg}^{2+}$ ions, indicating that $\mathrm{PV}$ is involved in a variety of cellular processes $(23,24)$. This suggests that in the MOB of SE rats, decreases in PV-positive interneurons alter not only GABAergic inhibitory functions but also various calcium-dependent cell signaling.

In a previous study of rat olfactory bulbs, Hwang et al. (9) reported that PV-positive interneurons had branched and well-developed processes with varicosities and spines like calbindin-positive interneurons. The well-developed processes of PV-positive interneurons have much denser connections with mitral cells than those between granule cells and mitral cells (14). However, we confirmed that PV immunoreactivity and dendritic density was markedly decreased following SE. Oxidative stress and neuroinflammation induced by SE can result in neuronal loss in the olfactory bulb, which decreased dendritic density in this study. In contrast, some studies have suggested that calcium signals are associated with the growth and branching of dendrites (25). Therefore, we can consider a correlation between decreased dendritic density and the roles of PV. Morphological changes in the dendritic processes are closely related to synaptic plasticity $(26,27)$ and we observed decreased dendritic density in the MOB of the SE rats. Decreases in the dendritic density of PV-positive interneurons important for odor tuning support a functional loss of olfaction.

In the present study, we confirmed morphologically that PV-positive interneurons were detected in the EPL and GL of the rat $M O B$ and that pilocarpine-induced SE resulted in neurodegeneration and a decrease in PV- and NeuN-positive neurons. In the $\mathrm{MOB}$, the reciprocal interaction of PV-positive interneurons with mitral cells is crucial for odor tuning (14) and the roles of PV for modulating calcium levels are essential for synaptic transmission and various neuronal function. Thus, the SE-induced changes in PV expression in the MOB strongly support the loss of olfaction seen in epilepsy patients.

\section{MATERIALS AND METHODS}

\section{Experimental animals}

All experiments utilized the progeny of Sprague-Dawley rats obtained from the Experimental Animal Center, Soonchunhyang University (Cheonan, South Korea). All animals were provided with a commercial diet and water ad libitum under controlled temperature, humidity and lighting conditions (light/dark cycle $12: 12$, and $22 \pm 2^{\circ} \mathrm{C}, 55 \pm 5 \%$ ). All animal protocols were approved by the Administrative Panel on Laboratory Animal Care of Soonchunhyang University (No. SCH16-0051). All possible efforts were made to avoid animal suffering and minimize the number used in the experiments.

\section{Seizure induction}

The SE rat model was induced by treatment with pilocarpine as previously described (28). The rats were pretreated with lithiumchloride before pilocarpine to ensure a high survival rate. Twenty hours after lithium-chloride $(127 \mathrm{mg} / \mathrm{kg}$, i.p.; Sigma-Aldrich, St. Louis, MO, USA) treatment, scopolamine was injected (2 mg/ kg, i.p.; Sigma-Aldrich) 30 min prior to pilocarpine $(30 \mathrm{mg} / \mathrm{kg}$, i.p.; Sigma-Aldrich) treatment, which induces SE. Two hours following pilocarpine treatment, diazepam was injected for depression. The seizures were classified into 5 grades according to behavioral characteristics (28), and the SE animals used in the study, consistently exhibited 4 or 5-grade seizures until they were injected with diazepam. For the study, the animals were divided into three groups; the control group, and groups at six hours and three months after SE.

\section{Fluoro-jade b (FJB) staining}

To identify degeneration, FJB staining was performed (29). Each section of the $M O B$ was mounted on gelatin-coated slides and air-dried overnight. The slides were immersed in $1 \% \mathrm{NaOH}$ in $80 \%$ alcohol for $5 \mathrm{~min}, 2 \mathrm{~min}$ in $70 \%$ alcohol, and $2 \mathrm{~min}$ in distilled water. Then, the slides were transferred to a $0.06 \%$ potassium permanganate solution for 10 minutes. The slides were washed in distilled water for 2 minutes. The staining 
solution was prepared from a $0.01 \%$ stock solution of fluorojade $b$ (Millipore, Burlington, MA, USA) in $0.1 \%$ acetic acid. The slides were incubated in a final concentration of $0.0004 \%$ for $20 \mathrm{~min}$, then dried for 1 hour. After drying, the slides were rinsed $1 \mathrm{~min}$ in xylene. The slides were mounted in mounting medium $4^{\prime}, 6$-diamidine-2' -phenylindole dihydrochloride (DAPI) (Vector Laboratories, Burlingame, CA, USA). All images were captured using a model Fluoview FV10i and FV10i software (Olympus, Japan).

\section{Immunohistochemistry and double immunofluorescence}

The anesthetized animals (urethane $1.5 \mathrm{~kg} / \mathrm{kg}$, i.p.; SigmaAldrich) were perfused transcardially with phosphate-buffered saline (PBS), followed by $4 \%$ paraformaldehyde in $0.1 \mathrm{M}$ phosphate-buffer (PB) ( $n=5$ in each group). The brains were removed and post-fixed in the same fixative for $4 \mathrm{~h}$, and rinsed in $\mathrm{PB}$ containing $30 \%$ sucrose at $4^{\circ} \mathrm{C}$ for 2 days. Thereafter, the tissues were frozen and sectioned with a cryostat at $30 \mu \mathrm{m}$ thickness and consecutive sections were collected in six-well plates containing PBS. The sections were incubated with primary rabbit anti-PV antibody(1:10000, Swant, Switzerland) in PBS containing $0.3 \%$ Triton X-100 overnight at room temperature. The sections were washed 3 times for $10 \mathrm{~min}$ with PBS, incubated sequentially in biotinylated goat anti-rabbit IgG (1:200, Vector Laboratories) and ABC complex (Vector Laboratories). The sections were visualized with 3,3' -diaminobenzidine (DAB) in $0.1 \mathrm{M}$ Tris buffer and mounted on gelatin-coated slides. The immunoreactions were observed using DMRB microscope (Leica, Germany) and the images were captured using a model DP72 digital camera and DP2-BSW microscope digital camera software (Olympus, Japan). To establish the specificity of the immunostaining, a negative control test was carried out with pre-immune serum instead of the primary antibody. The negative control resulted in the absence of immunoreactivity in any structures. To identify the morphological changes induced by SE in the same MOB tissue, double immunofluorescence staining for PV/NeuN and PV/MAP2 was performed. The MOB tissues were incubated overnight at room temperature in a mixture of rabbit anti-PV IgG $(1: 5,000$, Swant)/mouse anti-NeuN IgG (1:50, Millipore) and rabbit anti-PV IgG (1: 5,000, Swant)/mouse anti-MAP2 IgG (1:300, Millipore). After washing three times for 10 min with PBS, the sections were incubated in a mixture of Cy2- and Cy3-conjugated secondary antisera (1:200, Jackson Immunoresearch, Baltimore, PA, USA) for $1 \mathrm{~h}$ at room temperature. The sections were mounted with DAPI (Vector). All images were captured using a model Fluoview FV10i and FV10i software (Olympus, Japan).

\section{Western blot analyses}

To quantify the expression of PV following pilocarpine-induced status epilepticus (SE), the rats ( $n=5$ per group) were euthanized with urethane $(1.5 \mathrm{~kg} / \mathrm{kg}$, i.p.; Sigma-Aldrich). The olfactory bulbs were quickly removed and homogenized in a buffer, as previously described (30). The protein-transferred nitrocellulose membranes (Pall Crop, East Hills, NY, USA) were incubated with rabbit anti-PV IgG $(1: 10,000$, Swant Switzerland) and the data were normalized compared to the $\beta$-actin levels as described in a previous study (30).

\section{Quantification of data and statistical analysis}

The cell counts were performed by two different investigators who were blinded to the classification of the tissues. Each image was normalized by adjusting the black and white range of the image using Adobe PhotoShop v. 8.0. Thereafter, 10 areas per rat $\left(250 \times 250 \mu \mathrm{m}^{2}\right.$ for each area) were selected and the intensity measurements were represented as the mean number of a 256-shade gray-scale (using NIH Image 1.59 software). The optical density values were corrected by subtracting the average background noise values obtained from five image inputs. All data obtained from the quantitative measurements were analyzed using an one-way analysis of variance (ANOVA) to determine the statistical significance. Bonferroni's test was used for post-hoc comparisons. P-values of $<0.01,0.05$, and 0.005 was considered statistically significant (28).

\section{ACKNOWLEDGEMENTS}

This work was supported by the Soonchunhyang University and a Basic Science Research Program (NRF-2017R1D1A1B05 036195) through the National Research Foundation of Korea funded by the Ministry of Science.

\section{CONFLICTS OF INTEREST}

The authors have no conflicting interests.

\section{REFERENCES}

1. Al-Mufti F and Claassen J (2014) Neurocritical care: status epilepticus review. Crit Care Clin 30, 751-764

2. French JA (2007) Refractory epilepsy: clinical overview. Epilepsia 48, 3-7

3. Henshall D (2007) Apoptosis signaling pathways in seizureinduced neuronal death and epilepsy. Biochem Soc Trans $35,421-423$

4. Wang L, Liu YH, Huang YG and Chen LW (2008) Timecourse of neuronal death in the mouse pilocarpine model of chronic epilepsy using Fluoro-Jade C staining. Brain Res J 1241, 157-167

5. Helmstaedter C and Kockelmann E (2006) Cognitive outcomes in patients with chronic temporal lobe epilepsy. Epilepsia 47, 96-98

6. Mehla J, Reeta K, Gupta P and Gupta YK (2010) Protective effect of curcumin against seizures and cognitive impairment in a pentylenetetrazole-kindled epileptic rat model. Life Sci 87, 596-603

7. Desai M, Agadi J, Karthik N, Praveenkumar S and Netto A (2015) Olfactory abnormalities in temporal lobe epilepsy. J Clin Neurosci 22, 1614-1618

8. Imai T (2014) Construction of functional neuronal cir- 
cuitry in the olfactory bulb. Semin Cell Dev Biol 35, 180-188

9. Hwang IK, Kim DS, Lee HY et al (2003) Age-related Changes of Parvalbumin Immunoreactive Neurons in the Rat Main Olfactory Bulb. Mol Cells 16, 302-306

10. Caillard O, Moreno H, Schwaller B, Llano I, Celio MR and Marty A (2000) Role of the calcium-binding protein parvalbumin in short-term synaptic plasticity. Proc Natl Acad Sci U S A 97, 13372-13377

11. Choi WS, Chun SY, Markelonis GJ, Oh TH and Oh YJ (2001) Overexpression of calbindin-D28K induces neurite outgrowth in dopaminergic neuronal cells via activation of p38 MAPK. Biochem Biophys Res Commun 287, 656661

12. Mattson MP (2007) Calcium and neurodegeneration. Aging Cell 6, 337-350

13. Kim J, Kwak S, Kim D et al (2006) Reduced calcium binding protein immunoreactivity induced by electroconvulsive shock indicates neuronal hyperactivity, not neuronal death or deactivation. Neuroscience 137, 317-326

14. Kato HK, Gillet SN, Peters AJ, Isaacson JS and Komiyama T (2013) Parvalbumin-expressing interneurons linearly control olfactory bulb output. Neuron 80, 1218-1231

15. Kovac S, Domijan A, Walker M and Abramov A (2014) Seizure activity results in calcium-and mitochondria-independent ROS production via NADPH and xanthine oxidase activation. Cell Death Dis 5, e1442

16. González-Reyes S, Santillán-Cigales JJ, Jiménez-Osorio AS, Pedraza-Chaverri J and Guevara-Guzmán R (2016) Glycyrrhizin ameliorates oxidative stress and inflammation in hippocampus and olfactory bulb in lithium/pilocarpine-induced status epilepticus in rats. Epilepsy Res 126, 126-133

17. Hummel T, Henkel S, Negoias S et al (2013) Olfactory bulb volume in patients with temporal lobe epilepsy. J Neurol 260, 1004-1008

18. Nguyen MQ and Ryba NJ (2012) A smell that causes seizure. PLoS One 7, e41899

19. Suzuki Y, Kiyokage E, Sohn J, Hioki H and Toida K (2015) Structural basis for serotonergic regulation of neural circuits in the mouse olfactory bulb. J Comp Neurol 523, 262-280

20. Egger V and Urban NN (2006) Dynamic connectivity in mitral cell-granule cell microcircuit. Semin Cell Dev Biol $17,424-432$

21. Isaacson JS and Strowbridge BW (1998) Olfactory reciprocal synapses: dendritic signaling in the CNS. Neuron 20, 749-761

22. Schwaller B, Meyer M and Schiffmann S (2002) 'New' functions for 'old'proteins: the role of the calcium-binding proteins calbindin D-28k, calretinin and parvalbumin, in cerebellar physiology. Studies with knockout mice. Cerebellum 1, 241-258

23. Schwaller B (2009) The continuing disappearance of "pure" Ca $2+$ buffers. Cell Mol Life Sci 66, 275-300

24. Yu L, Xu L, Xu M, Wan B, Yu L and Huang Q (2011) Role of $\mathrm{Mg} 2+$ ions in protein kinase phosphorylation: insights from molecular dynamics simulations of ATP-kinase complexes. Mol Simul 37, 1143-1150

25. Konur S and Ghosh A (2005) Calcium signaling and the control of dendritic development. Neuron 46, 401-405

26. Yuste R and Bonhoeffer T (2001) Morphological changes in dendritic spines associated with long-term synaptic plasticity. Annu Rev Neurosci 24, 1071-1089

27. Sjostrom PJ, Rancz EA, Roth A and Hausser M (2008) Dendritic excitability and synaptic plasticity. Physiol Rev 88, 769-840

28. Kim DS, Kim JE, Kwak SE et al (2008) Spatiotemporal characteristics of astroglial death in the rat hippocampoentorhinal complex following pilocarpine-induced status epilepticus. J Comp Neurol 511, 581-598

29. Schmued LC and Hopkinsa CJ (2000) Fluoro-Jade B: a high affinity fluorescent marker for the localization of neuronal degeneration. Brain Res J 874, 123-130

30. Jung HY, Kim DW, Nam SM et al (2017) Pyridoxine improves hippocampal cognitive function via increases of serotonin turnover and tyrosine hydroxylase, and its association with CB1 cannabinoid receptor-interacting protein and the CB1 cannabinoid receptor pathway. Biochim Biophys Acta Gen Subj 1861, 3142-3153 